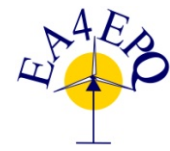

$19^{\text {th }}$ International Conference on Renewable Energies and Power Quality (ICREPQ'21)

Almeria (Spain), $28^{\text {th }}$ to $30^{\text {th }}$ July 2021

Renemable Energy and Pourer Quality. Fournal (RE\&PQJ)

ISSN 2172-038 X, Volume No.19, September 2021

\title{
Protection of Power Semiconductors in Inverters, using Fuses and their Coordination with the Protection Schemes of the Distribution System
}

\author{
J. C. Gómez, J. Vaschetti, M. Piumetto, J. Arcurio and C. Coyos \\ Department of Electricity \\ CIDTIEE, Technological National University \\ Maestro López esquina Cruz Roja Argentina, 5000, Córdoba (Argentine) \\ Phone/Fax number: +0054 358 4676171, e-mail: jcgomez@ing.unrc.edu.ar
}

\begin{abstract}
The integration of Distributed Energy Resources (DER) implies an important challenge for the protection of the distribution network, due to the incorporation of devices with less capacity to withstand normal disturbances in distribution systems, particularly for overcurrents and overvoltages. The increasingly widespread use of power electronics, incorporated in rectifiers and inverters, which due to its weakness requires highspeed protection devices, complicates coordination with the traditional protection of distribution systems. Converters (rectifiers and inverters) require ultra-fast protection against high current faults, conditions that currently only meet high breaking capacity fuses. The response characteristics of these ultra-fast fuses, oblige the professional in charge of the protection of the distribution system to have a deep knowledge about the behavior of the power electronics in the face of overcurrents, and to know also how the fuses, through the selection of its rated values and characteristic curves protects it. Coordination of the rating values and characteristics of these fuses with their counterparts (when they exist) of traditional protection devices is the main objective of this article. Particular terms are explained in detail, such as fault current asymmetry and its effect on the coordination of protections, specific energy, etc. It is concluded that deep knowledge of the dissimilar characteristics of power electronics devices and the usual devices of distribution networks, with regard to their protections, are essential to obtain a maximum use of the traditional scheme with the addition of DER.
\end{abstract}

Key words. Fuses, distribution, power electronics, ultra-fast protection.

\section{Introduction}

The integration of Distributed Energy Resources (DER) implies an important challenge for the protection of the distribution network, due to the incorporation of devices with less capacity to withstand normal disturbances in distribution systems, particularly in front of overcurrents and overvoltages. The increasingly widespread use of power electronics, incorporated in rectifiers and inverters, which due to its weakness requires high-speed protection devices, complicates coordination with the traditional protection of distribution systems. It should be remembered that the primary or "first" function of such devices is to protect the semiconductor, and secondly or "secondary" function, it must coordinate with the other protective devices. Power electronics (rectifiers and inverters) require ultra-fast protection with low values of overvoltage generated in the interruption process, against high intensity faults, conditions that currently only meet high breaking capacity fuses [1].

\section{Short Circuit Current and Fault Current Asymmetry}

Reference will only be made to cases with alternating current power supply, the situation in relation to direct current is analyzed in another article of this same Conference.

The study of the behavior against alternating current and unidirectional pulsation, controlled or not, is similar, but not the behavior on direct current due to the lack of natural passages through zero.

The current of a faulty circuit is that corresponding to a circuit with only resistors and inductances; capacitive values (distributed capacities) can be neglected due to their small value in distribution systems. Therefore, the analytical expression of this current has two components, called "direct current" because it attenuates in time in an exponential way (according to the time constant $\tau$ equal to the inductance / resistance ratio) without oscillating and the component of "alternating current" that oscillates with the network frequency $(\omega)$, without attenuation.

$\mathrm{i}=\sqrt{2} \mathrm{I}\left(\operatorname{sen}(\omega \mathrm{t}+\theta-\varphi)-\mathrm{e}^{-\mathrm{t} / \tau} \operatorname{sen}(\theta-\varphi)\right)$

In the equation there are two angles: $\varphi$ which corresponds to the power factor of the faulty circuit and $\theta$ which is the angle at which the fault starts, measured from the zero passage of the voltage, which is totally random. For this reason, the waveform of the fault current can be without a direct current component, if the disturbance occurs when the angles $\theta$ and $\varphi$ are equal, a so-called symmetric waveform; or totally asymmetric when the angular difference $\theta-\varphi$ is $90^{\circ}$. Figure 1 shows both components (in red and black, respectively) and the resulting one for the worst case. In the aforementioned figure, it can be seen that in the absence of the DC component, the current grows with the "speed" set by the amplitude and the 
network frequency. The random presence of the DC component can significantly change the growth "speed", a parameter that is of crucial importance in this application, where the protection must act in the first milliseconds of the failure. In the case of traditional equipment in distribution systems, their high robustness made this first part of the fault current uninteresting. The question that arises is how the fuse manufacturer informs the user of this particular behavior of the ultra-fast fuse, which was initially provided in the time-current characteristic curve, whose time axis started in $1 \mathrm{~ms}$, by means of the bifurcation of the curve for times less than 10 to $50 \mathrm{~ms}$, on two lines corresponding to symmetric and asymmetric current (maximum possible of normal circuits). Currently, it is reported with the specific energy value for times of 1.2 $\mathrm{ms}$; a value that can increase significantly if it is considered the $10 \mathrm{~ms}$, starting value of the time axis of the characteristic curves for traditional fuses in distribution systems [2].

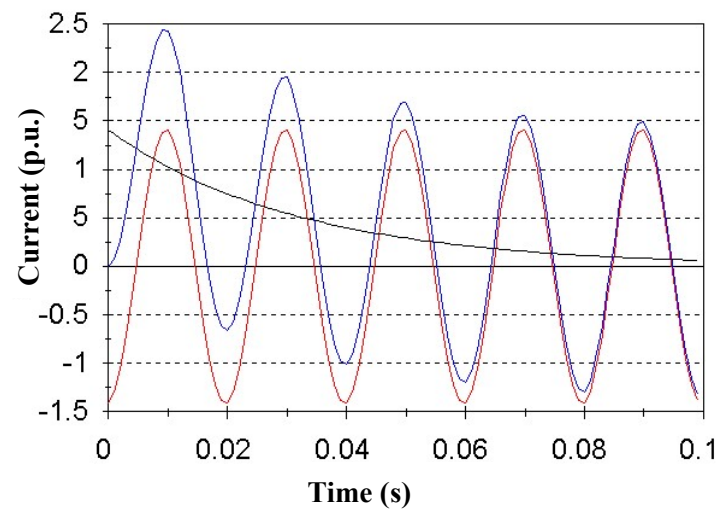

Figure 1, Components of direct, alternating and resulting fault current.

\section{Characteristics of Power Electronic Devices}

Today's semiconductors have the characteristic of being capable of conducting high load current values, further increased with the addition of "heat sinks" and forced cooling. Moreover, they are very weak to overcurrents and overvoltages. In general, it can be said that a semiconductor supports twice its nominal current for only a few milliseconds (10 to 20), as opposed to, for example, a transformer, which, starting from room temperature, supports twice the load for almost half an hour. The information of this semiconductor capacity or ability, is one of the parameters that must inevitably be controlled by the fuse. Minor overloads can be "cut off" by the control circuit, acting on the gate. There are power electronics devices that can "turn off" high currents, generating the necessary overvoltage to reverse the increasing trend of fault current, called limiting overvoltage (solid state switch) [3]. Such equipment are not yet widespread, but they will be seen with increasing frequency in the coming years.

There are numerous articles and manufacturer application guides regarding how to select external inverter protections, published mainly by fuse and inverter manufacturers, which in the case of photovoltaic sources has led to the IEC standardization of a new class of fuses called gPV. One of the main characteristics of these fuses is the ability to act against the low fault currents supplied by the photovoltaic panels $[4,5]$.

Power semiconductors are very sensitive to overvoltages, in three of their characteristics: growth speed, peak value and duration. These limits are normally reported in the semiconductor catalogs or technical sheets, values that must be compared with the homologues generated by the protection during its operation. In the event that a traditional distribution type fuse is installed near the inverter's alternating current terminals, an element that can generate high overvoltages (usually greater than 2,000 V), gaseous discharge type overvoltage protectors (varistor and avalanche diode) must be installed, devices that are normally installed by the possibility of being reached by the energy of atmospheric discharges $[4,6]$. The energy of the fuse operation overvoltage is very high since its duration is of the order of milliseconds, as opposed to the microseconds duration of lightning [1].

\section{Characteristics of the Ultra-fast Fuses}

In general, fuses in distribution systems have a very high breaking capacity (maximum current that they are capable of interrupting without external manifestation), normally reaching $100 \mathrm{kA}$, but they have limitations in the interruption of low currents or slight overloads. This limitation was resolved through the addition of a low melting point metal alloy deposit, which allowed the fuses to interrupt currents as low as $30 \%$ above the rated, an addition known as "M-effect". Fuses with M-effect are called general purpose or extended field, identifying them with a letter $g$ (general use), those that do not have this addition are identified with the letter a (accompaniment or support). The original German standard, standardized a group of fuses designed especially for particular applications, calling the fuses with two (or three in one case) letters, the first a or $\mathrm{g}$ based on what has been explained and the second (capital letter) that indicates the application: $\mathrm{L}$ (or $\mathrm{G}$ in the IEC standardization) for general applications, especially protection of lines and cables, Tr for transformers, $M$ for motors, B for mining applications and $\mathrm{R}$ for semiconductors. Thus, the aR and gR classes appear in contrast to those traditionally used in distribution systems, $\mathrm{gL}$ (or $\mathrm{gG}$ ) and $\mathrm{gTr}$. The characteristic curves of each fuse type are dissimilar and are strictly suitable for the protection of each particular device. The utilities professionals were normally familiars with the fuses of classes' $\mathrm{gL}$ (or $\mathrm{gG}$ ) and in some cases also with the $\mathrm{gTr}$, which are rather simpler than the characteristics of $\mathrm{aR}$ and $\mathrm{gR}$ classes.

The response characteristics of these ultra-fast fuses, oblige the professional in charge of the protection of the distribution system to have a deep knowledge about the behavior of the power electronics in the face of overcurrents, and to know how the fuses, through the selection of its rated values and characteristic curves protects it.

The operation of the fuse is divided into two periods, the first called pre-arc, a period during which the fuse element raises its temperature due to the Joule heat generated by the fault current being circulated, until 
reaching the melting temperature, during this time the current conduction is carried out with a fuse element in solid or liquid state. When the melting temperature is reached, the solid conduction ends, beginning the arc period, which ends when the current circulation is finally extinguished. Both periods, pre-arc and arc, have their durations, passing energies, maximum current and voltage, and other characteristics. In a simplified way, if it is desired that the fuse withstand the overcurrent without being affected, the energy that passes through it must be less than that of the pre-arc, taking into account a small tolerance (tolerance corresponding to the manufacturing process of the device). If, on the other hand, it is wanted to limit the energy allowed to pass through the fuse, the prearc energies plus the arc including a small tolerance, must be controlled [1].

Due to the complexity of this application, the fuses must have more complete operating information, mainly provided in the form of characteristic curves. Industrial type fuses, in addition to their nominal characteristics, report their operation based on three characteristic curves, Time - Current curve, Pass Through Current - Prospective Current graph and Specific Energy $\left(\mathrm{I}^{2} \mathrm{t}\right)$ - Rated Current graph [2].

As the ultra-fast fuses are designed with a high restriction ratio (ratio between the largest and smallest section of the fuse element), the concept of adiabatic operation is limited to lower values of pre-arc time, with maximum values of the order of $1 \mathrm{~ms}$.

As this type of fuses is the most demanded from the point of view of design and construction, they are affected by parameters such as ambient temperature on load capacity and altitude with respect to sea level on breaking capacity and working voltage [7].

Furthermore, in contrast to the devices of classes gL (or $\mathrm{gG}$ ) and $\mathrm{gTr}$, in the fuses classes $\mathrm{gR}$ and $\mathrm{aR}$, the applied voltage and the power factor (or time constant depending on the case) have a considerable influence on their behavior, modifying or affecting the traditional characteristic parameters.

Until now, the restriction relationship has been mentioned, but nothing has been said about the form of such a restriction. The shape of the restriction is crucial in the way in which the operating overvoltage is generated, which is a critical element due to the high sensitivity of semiconductors to it. If the restriction is pronounced and long, a very good control of the specific energy is obtained, but the growth slope of the generated overvoltage, dangerously increases. Therefore, gradual growth restrictions are used, which offers a compromise solution between the specific energy and the slope of the surge. Another constructive difference lies in the use of solidified quartz sand in order to strictly guarantee the size of the chamber where the arc is extinguished, and thus the overvoltage limited.

\section{Protector - Protected Coordination}

The protective-protected coordination task is based on confirming that the stress (or energy) allowed to pass through the protector (fuse in this study) does not exceed the energy supported without damage by the protected device in series and also that the overvoltages generated in the limiting operation case do not damage the devices connected in parallel with the protected one. In other words, the coordination work is a simple comparison of the parameters of both devices.

With low and medium overcurrents, the time-current characteristic curves must be directly compared, on the other hand, for high values of fault current, it is necessary to refer to the specific energy or $\mathrm{I}^{2} \mathrm{t}$, which is no longer constant for times as short as $1 \mathrm{~ms}$ ( power factor and connection angle determine the worst conditions). In semiconductor catalogs, the specific supported energy value is specifically cited, called " $\mathrm{I}^{2} \mathrm{t}$ for fuse" or "I $\mathrm{I}^{2} \mathrm{for}$ fusing".

The total specific energy, due to the effect on that corresponding to the arc period, must be modified based on the working voltage. Situation that to a lesser extent was also present in the case of industrial fuses, since for example NH (Niederspannung Hochleistung, meaning low voltage high power in German) type fuses have a design voltage of $500 \mathrm{~V}$ and are normally used in distribution systems whose usual voltage value is 380 / $400 \mathrm{~V}$. This modification is carried out through correction factors, which are very significant in the case of fuses for power electronics, caused by the high restriction ratio required.

As the power electronics are very sensitive to overvoltages, the maximum value of the overvoltage generated in the interruption (usually with the current I1) is reported as a function of the working voltage, a subject that is not so demanding for the industrial fuse, whose limit is specified in the corresponding IEC standard in table form, depending on the working voltage.

If the study is carried out on the DC side of the inverter, the working voltage must be modified as a function of the time constant of the faulty circuit.

Variations in power losses as a function of the nominal current are also taken into account due to the restrictions on heat extraction due to the low space availability.

When the incorporation of power electronics in distribution systems refers to the use of inverters for the injection of renewable energy mainly generated in direct current, such inverters internally include protection, so that protection against overloads is carried out by controlling the current conduction and protecting against short circuits by fuses (internal). This protection must be complemented by external protective devices, in order to avoid damage to the inverter by the energy released from the grid (in alternating current) and from the source (in alternating and direct current). In addition, it is very likely that there are elements for energy storage, on the alternating or DC sides, which considerably increase the possible amount of energy released.

\section{Coordination of Ultra-fast Fuses with other Protective Devices}

An initial factor to take into account refers to the fact that the normal protective devices of the distribution system are of the so-called full field, that is, they are capable of detecting and interrupting any fault current value (higher than its nominal current). On the other hand, most of the 
fuses used to protect semiconductors are class a (that is, with a field limited to rigorous overcurrents, more than 6 to 8 times the rated current). Although it is true that there are ultra-fast class $\mathrm{g}$ fuses, their application is still low because after cutting a slight overload (normally quite frequent) the replacement of the fuse is required, which is expensive, having been able to interrupt that fault current in another way without sacrificing the fuse.

The traditional coordination between protective devices against overcurrent is based on the comparison of the characteristic time - current curves, which here is applicable only in the validity zone of the homologous curve of the semiconductor, which have very different slopes, since the device of the distribution system is designed for equipment with high overload capacity. For high fault currents, the ultra-fast fuse will always operate before the distribution system device, although if they are needed, they can be coordinated based on the $I^{2} t$ values affected by the corresponding correction factors [8].

In this coordination of protection devices analysis, an important difference is introduced compared to traditional studies, which is the variability of the fault current. This variability is due to the action of the inverter control or intrinsic characteristics of the source, as is the case of photovoltaic generation, whose short-circuit current is only 15 or $20 \%$ higher than the rated load current [4]. This variability and current limitation modifies the protection selection criteria that based the detection of the fault and the speed of operation on the magnitude of the overcurrent that crosses it. The traditional time-current curves were designed to be used with currents of constant value (amplitude or rms value), so it is necessary to complement the coordination work using the curves of operation time as a function of the specific energy, which allows the analysis for variable currents and even for devices not strictly in series [9].

The coordination of the values and nominal characteristics of the ultra-fast fuses with the counterparts (when they exist) of the traditional protection devices is somewhat more complex, requiring more information about the devices involved and more knowledge of who is conducting the study.

\section{Conclusion}

It is concluded that in-depth knowledge of the dissimilar characteristics of power electronics devices and the usual devices of distribution networks, with regard to their protections, are essential to obtain maximum use of the traditional scheme with the addition of distributed renewable sources.

\section{References}

[1] Gómez, J. C., Fusibles eléctricos: aplicaciones prácticas y su justificación teórica, EDIGAR, Buenos Aires, Argentina, 2012.

[2] Jacks, E., 1975, High rupturing capacity fuses, E. \& F.N. Spon Ltd., London, England. 1975.

[3] Rodrigues, R., Yu Du, Antoniazzi, A., Cairoli, P., A Review of Solid-State Circuit Breakers, IEEE Transactions on Power Electronics, Vol. 36, Issue 1, 2021.

[4] Photovoltaic System Protection Application Guide, Cooper Bussmann Ltd, United Kingdom, 2012.

[5] IEC 60269-6 ed. 1.0, Low-voltage fuses - Part 6: Supplementary requirements for fuse-links for the protection of solar photovoltaic energy systems, 2010.

[6] IEC 60269-1, ed. 4.1, Low-voltage fuses - Part 1: General requirements, 2009.

[7] Wright, A. Newbery, P.G., Electric Fuses, Second edition, Peter Peregrinus Ltd., London, 1984.

[8] Bessei, H., Manual de Fusibles, NH/HH, Recycling e.V., Lappersdorf, Alemania, 2012.

[9] Nesci, S., Gómez, J. C., Sanchez, L., Rovere, H., Overcurrent coordination by the application of the specific energy methodology on distribution systems having distributed generation, $7^{\text {th }}$ International Youth Conference on Energy 2019, 3 to 6 July, 2019, Bled, Slovenia. 